\title{
A CHARACTERIZATION OF THE WEAKLY CONTINUOUS POLYNOMIALS IN THE METHOD OF COMPENSATED COMPACTNESS
}

\author{
ROBERT C. ROGERS AND BLAKE TEMPLE
}

\begin{abstract}
We present a sufficient condition for weak continuity in the method of compensated compactness. The condition links weak continuity to the structure of the wave cone and the characteristic set for polynomials of degree greater than two. The condition applies to all the classical examples of weakly continuous functions and generalizes the Quadratic Theorem and the Wedge Product Theorem. In fact, the condition reduces to the LegendreHadamard Necessary Condition when the polynomial is quadratic, and also whenever a certain orthogonality condition is satisfied. The condition is derived by isolating conditions under which the quadratic theorem can be iterated.
\end{abstract}

1. Introduction. In this paper we present a pointwise condition, sufficient to guarantee weak continuity in the method of compensated compactness. Our condition, which is stated in terms of the wave cone and characteristic set of the first-order linear differential constraints in the method, generalizes both the Wedge Product Theorem $[\mathbf{4}, \mathbf{7}]$ and the Quadratic Theorem $[\mathbf{5}, \mathbf{6}]$ in a natural way.

Here we restrict our attention to sequences of quadratic maps (analogous to sequences of wedge products) and corresponding sequences of differential operators (analogous to the exterior derivative). We consider polynomials obtained by iterating these quadratic maps. Our main result is a sufficient condition for the weak continuity of such polynomials on sequences constrained by the differential operators. We show that the resulting condition is sharp in the sense that it is the best such condition that takes account only of bounds on the derivatives and not more subtle constraints imposed on the oscillations by the polynomial. Moreover, all smooth weakly continuous functions are generated by polynomials (cf. [1]), and every polynomial can be obtained by iterating quadratic maps. (For instance, the $r$ th Fréchet derivative of an $r$ th-order polynomial is a constant $r$-linear form which we can iterate "slot-by-slot".) Thus our condition provides a practical method of testing functions for weak continuity.

We do not presently know whether all weakly continuous polynomials converge as a result of our condition, but it is interesting to note that a necessary condition of Murat [3], the best local necessary condition of which we are aware, is stated

Received by the editors September 20, 1987.

1980 Mathematics Subject Classification (1985 Revision). Primary 46A50; Secondary 35A30, 53A45.

First author partially supported by the National Science Foundation under Grants No. DMS8521687 and DMS-8620303.

Second author partially supported by the National Science Foundation under Grant No. DMS8613450 . 
in terms of multilinear forms and iterates of quadratic maps. Our condition is nominally stronger than Murat's, but we know of no function that satisfies one but not the other. As further evidence of the generality of our condition we note that it reduces to the Legendre-Hadamard necessary condition when the polynomial is quadratic and also when a certain orthogonality condition is satisfied.

The condition we derive also clarifies the relationship between the directions in the wave cone (associated with the dependent variables of the weakly convergent sequence) and the characteristic directions (associated with the independent variables of the sequence). The Legendre-Hadamard condition involves only the wave cone, but for degree greater than two, the coupling of these directions to the characteristic directions is crucial. Murat's condition takes account of this coupling in a different way.

Our point of view here is to consider the set of rank-1 matrices obtained as the tensor product of elements in the wave cone with the characteristic directions. Our theorem is obtained by studying natural orthogonality conditions that arise in this space.

ACKNOWLEDGMENT. The second author is indebted to Professor Lars Hörmander for showing him the proof of Lemma (3.1). This author would also like to thank Professor Hörmander for his gracious hospitality during a stimulating visit to the Mittag-Lefler Institute in April and May of 1986. The authors would like to thank Professor Evan Fletcher for locating several errors in the original manuscript.

2. A sufficient condition for weak continuity. Let $\left\{U^{\varepsilon}\right\}_{\varepsilon \rightarrow 0}$ denote a sequence of functions

$$
U^{\varepsilon}: \mathbf{R}^{I} \rightarrow \mathbf{R}^{J}
$$

that satisfy

$$
\left\|U^{\varepsilon}\right\|_{\infty}<\text { Const }
$$

$$
\sum_{i, j} A_{i j}^{k} \frac{\partial U_{j}^{\varepsilon}}{\partial x_{i}} \in \text { compact set in } H_{\mathrm{loc}}^{-1}\left(\mathbf{R}^{I}\right), \quad k=1, \ldots, K \text {. }
$$

Here $\|\cdot\|_{\infty}$ denotes the $L^{\infty}\left(\mathbf{R}^{I}\right)$ norm, $i$ is summed from 1 to $I, j$ from 1 to $J$, and $A_{i j}^{k}$ are given constants. We adopt the convention of using subscripts to denote components of vectors or matrices, superscripts to denote position in a sequence or collection. Inequality (2.2) implies that by passing to a subsequence we can assume that

$$
U^{\varepsilon} \stackrel{*}{\rightarrow} \bar{U} \quad \text { in } L^{\infty}\left(\mathbf{R}^{I}\right)
$$

i.e.,

$$
\int_{\mathbf{R}^{I}} U^{\varepsilon} \phi \rightarrow \int_{\mathbf{R}^{I}} \bar{U} \phi
$$

for all $\phi \in L^{1}\left(\mathbf{R}^{I}\right)$. Here we address the problem of determining the functions $f$,

$$
f: \mathbf{R}^{J} \rightarrow \mathbf{R},
$$

that are weakly continuous under the differential constraints (2.4); i.e., functions $f$ for which

$$
f\left(U^{\varepsilon}\right) \stackrel{*}{\rightarrow} f(\bar{U}) \quad \text { in } L^{\infty}\left(\mathbf{R}^{I}\right)
$$

for all sequences satisfying (2.1)--(2.4). 
For example, if (2.3) implies that all derivatives of $\left\{U^{\varepsilon}\right\}$ lie in a compact set in $H_{\text {loc }}^{-1}\left(\mathbf{R}^{I}\right)$ then by compact imbedding $U^{\varepsilon} \rightarrow \bar{U}$ (strongly) in $L_{\text {loc }}^{2}\left(\mathbf{R}^{I}\right)$. In this case all continuous functions are weakly continuous. At the other extreme, when (2.3) is vacuous, then only the affine functions of $U$ are weakly continuous. To see this note that if $\lambda \in \mathbf{R}^{J}$, and $x \cdot \xi$ denotes the ordinary dot product of $x$ and $\xi$ in $\mathbf{R}^{I}$, then

$$
U^{\varepsilon}=\lambda g\left(\frac{x \cdot \xi}{\varepsilon}\right)-\frac{\lambda}{|P|} \int_{P} g(\xi) d \xi
$$

for any function $g: \mathbf{R} \rightarrow \mathbf{R}$ of period $P$. Our problem, then, is to characterize the weakly continuous functions when (2.3) is neither complete nor vacuous.

Information on the oscillations allowed by (2.3) is contained in the following sets:

$$
\mathscr{V}=\left\{(\xi, \lambda) \in \mathbf{R}^{I} \times \mathbf{R}^{J}: \sum_{i, j} A_{i j}^{k} \xi_{i} \lambda_{j}=0, k=1, \ldots, K\right\},
$$

We identify $\mathscr{V}$ with the set of rank-1 tensors determined by $(\xi, \lambda) \in \mathscr{V}$; i.e.,

$$
\mathscr{V} \equiv\left\{\xi \otimes \lambda \in \mathscr{L}\left(\mathbf{R}^{J}, \mathbf{R}^{I}\right): \sum_{i, j} A_{i j}^{k} \xi_{i} \lambda_{j}=0, k=1, \ldots, K\right\},
$$

where $\xi \otimes \lambda$ denotes the tensor product of $\xi$ and $\lambda$ : the $I \times J$ matrix with components $\xi_{i} \lambda_{j}$.

Observe that

$$
U^{\varepsilon}(x)=\lambda g\left(\frac{x \cdot \xi}{\varepsilon}\right)
$$

solves

$$
\sum_{i, j} A_{i j}^{k} \frac{\partial U_{j}^{\varepsilon}}{\partial x_{i}}=0, \quad k=1, \ldots, K,
$$

for each $(\xi, \lambda) \in \mathscr{V}$, and each $g: \mathbf{R} \rightarrow \mathbf{R}$ periodic of period $P$. We call (2.12) a rank-1 oscillator because the derivative of $U^{\varepsilon}$ is everywhere a multiple of the rank-1 tensor $\xi \otimes \lambda \in \mathscr{V}$.

Our sufficient condition is modeled after the following theorem.

Theorem (WEdGe Product TheOREM). For each $l=0, \ldots, L$, let $\omega^{l, \varepsilon}$ denote a differential form on $\mathbf{R}^{I}$ of degree $s^{l}$. Assume that

$$
\begin{gathered}
\left\|\omega^{l, \varepsilon}\right\|_{\infty}<\text { Const ., } \\
\mathbf{d} \omega^{l, \varepsilon} \in \text { compact set in } H_{\text {loc }}^{-1}\left(\mathbf{R}^{I}\right),
\end{gathered}
$$

and

$$
\omega^{l, \varepsilon} \stackrel{*}{\rightarrow} \bar{\omega}^{l} \quad \text { in } L^{\infty}\left(\mathbf{R}^{I}\right) .
$$


Then

$$
\omega^{0, \varepsilon} \wedge \cdots \wedge \omega^{L, \varepsilon} \stackrel{*}{\rightarrow} \bar{\omega}^{0} \wedge \cdots \wedge \bar{\omega}^{L} \quad \text { in } L^{\infty}\left(\mathbf{R}^{I}\right) .
$$

Here $\mathbf{d}$ denotes the exterior derivative and $\wedge$ the wedge product.

We generalize the Wedge Product Theorem by replacing wedge products with arbitrary quadratic maps and exterior derivatives with arbitrary first-order linear differential operators: Let $U^{\varepsilon}$ have the decomposition

$$
U^{\varepsilon}=\left(u^{0, \varepsilon}, \ldots, u^{L, \varepsilon}\right)
$$

and assume that

$$
\begin{gathered}
u^{l, \varepsilon}: \mathbf{R}^{I} \rightarrow \mathbf{R}^{J_{l}}, \quad l=0, \ldots, L, \\
\left\|u^{l, \varepsilon}\right\|_{\infty} \leq \text { Const . }, \quad l=0, \ldots, L
\end{gathered}
$$

$(2.3)_{l} \sum_{i, j} A_{i j}^{l k} \frac{\partial u_{j}^{l, \varepsilon}}{\partial x_{i}} \in$ compact set in $H_{\mathrm{loc}}^{-1}\left(\mathbf{R}^{I}\right), \quad k=1, \ldots, K^{l}, l=0, \ldots, L$.

and

$$
\left(u^{0, \varepsilon}, \ldots, u^{L, \varepsilon}\right) \stackrel{*}{\rightarrow}\left(\bar{u}^{0}, \ldots, \bar{u}^{L}\right) .
$$

Here $i$ is summed from 1 to $I ; j$ from 1 to $J_{l}$. We let $J=\sum_{l=0}^{L} J_{l}, K=\sum_{l=0}^{L} K_{l}$; and we let $(2.3)$ represent the collection of statements $(2.3)_{0},(2.3)_{1}, \ldots,(2.3)_{L}$. In this way $U^{\varepsilon}$ satisfies (2.1)-(2.4).

We now consider the following iterative method for generating polynomials in $U^{\varepsilon}$. Let

$$
Q^{l}: \mathbf{R}^{M_{l}} \times \mathbf{R}^{J_{l}} \rightarrow \mathbf{R}^{M_{l+1}}, \quad l=1, \ldots, L ;
$$

denote a sequence of quadratic maps, where $\left\{M_{l}\right\}_{l=1}^{L+1}$ is a set of positive integers with $M_{1}=J_{0}$ and $M_{L+1}=1$, and where $Q^{l}(v, w)$ is homogeneous of degree 1 in $v$ and $w$ respectively. Define the polynomial $P(U) \equiv P^{L}\left[u^{0}, \ldots, u^{L}\right]$ by the following recursion formula:

$$
\left\{\begin{array}{l}
P^{1}\left[u^{0}, u^{1}\right] \equiv Q^{1}\left(u^{0}, u^{1}\right) \\
P^{l}\left[u^{0}, \ldots, u^{l}\right] \equiv Q^{l}\left(P^{l-1}\left[u^{0}, \ldots, u^{l-1}\right], u^{l}\right), \quad l=2, \ldots, L
\end{array}\right.
$$

Our condition for weak continuity of $P$ depends on the interplay between the characteristic sets

$$
\mathscr{V}_{l}=\left\{\xi \otimes \lambda \in \mathscr{L}\left(\mathbf{R}^{J_{l}}, \mathbf{R}^{I}\right): \sum_{i, j} A_{i j}^{l k} \lambda_{j} \xi_{i}=0, k=1, \ldots, K^{l}\right\},
$$

and the interaction sets defined for each $l=2, \ldots, L$ and $m=0, \ldots, l-1$ by

$$
\begin{aligned}
& \mathscr{W}_{l}^{m} \equiv\left\{\xi \otimes \bar{\lambda} \in \mathscr{L}\left(\mathbf{R}^{M_{l}}, \mathbf{R}^{I}\right): \bar{\lambda}=P^{l-1}\left[v^{0}, \ldots, v^{m-1}, \lambda^{m}, v^{m+1}, \ldots, v^{l-1}\right]\right. \\
& \left.\quad \text { for some } \xi \otimes \lambda^{m} \in \mathscr{V}_{m}, v^{n} \in \mathbf{R}^{J_{n}}, 0 \leq n \leq l-1, n \neq m\right\},
\end{aligned}
$$

and

$$
\mathscr{W}_{l}= \begin{cases}\mathscr{V}_{0}, & l=1, \\ \bigcup_{m=0}^{l-1} \mathscr{W}_{l}^{m}, & l=2, \ldots, L .\end{cases}
$$

The condition is given as follows. 
THEOREM (SUfF). Let $P$ be defined by (2.14). Then if

$$
Q^{l}(\bar{\lambda}, \lambda)=0, \quad l=1, \ldots, L,
$$

whenever $\xi \otimes \bar{\lambda} \in \operatorname{span} \mathscr{W}_{l}$ and $\xi \otimes \lambda \in \mathscr{V}_{l}$ for the same $\xi \in \mathbf{R}^{I}$, then we have $P^{L}\left[u^{0, \varepsilon}, \ldots, u^{L, \varepsilon}\right] \rightarrow P^{L}\left[\bar{u}^{0}, \ldots, \bar{u}^{L}\right]$, for every sequence satisfying $(2.1)_{l}-(2.4)_{l}, l=$ $0, \ldots, L$.

The proof of Theorem (Suff) is postponed until $\S 3$.

We now examine Theorem (Suff) in the context of other conditions on weak continuity. We first recall that a function is weakly continuous if and only if it is $A$-quasiconvex:

Definition. A function $f: \mathbf{R}^{J} \rightarrow \mathbf{R}$ is said to be $A$-quasiconvex if

$$
\int_{D} f(\mu+\varsigma(x)) d x \geq \int_{D} f(\mu) d x=f(\mu) \text { meas } D
$$

for every $\mu \in \mathbf{R}^{J}$, for every hypercube $D \subset \mathbf{R}^{I}$, and for every $\varsigma: D \rightarrow \mathbf{R}^{J}$ such that $\varsigma \in L^{\infty}(D), \int_{D} \zeta(x) d x=0$, and $\sum_{i, j} A_{i j}^{k}\left(\partial \zeta_{j} / \partial x_{i}\right)=0$ in $W^{-1, \infty}(D), k=$ $1, \ldots, K$.

This condition was first isolated by Morrey [2] in the special case of the calculus of variations. While this completely characterizes weak continuity, it is a nonlocal condition and accordingly difficult to verify. Thus, local conditions are still of interest.

The first such condition we state is a generalization of the familiar necessary condition of Legendre-Hadamard from the calculus of variations.

THEOREM. If a function $f$ is weakly continuous, then it satisfies

$$
f(\mu+t \lambda) \text { is affine in } t, \quad \forall \mu \in \mathbf{R}^{J}, t \in \mathbf{R}, \lambda \in \Lambda .
$$

This result follows directly from (2.12) and (2.13).

The following well-known result of Tartar $[\mathbf{5}, \mathbf{6}]$ states that in the case of quadratic polynomials condition (L-H) is both necessary and sufficient for weak continuity.

THEOREM (TA). Let $Q: \mathbf{R}^{J} \rightarrow \mathbf{R}^{m}$ quadratic in $U^{\varepsilon}$. Then

$$
Q\left(U^{\varepsilon}\right) \stackrel{*}{\rightarrow} Q(\bar{U}) \quad \text { in } L^{\infty}\left(\mathbf{R}^{I}\right)
$$

for all $U^{\varepsilon}$ satisfying $(2.1)-(2.4)$ if and only if

$$
Q(\lambda)=0
$$

for every $\lambda \in \Lambda$.

Well-known counterexamples show that $(\mathrm{L}-\mathrm{H})$ is not sufficient for degree higher than two.

A stronger necessary condition of Murat [3] applies to higher order polynomials and is written in terms of multilinear forms (as is Theorem (Suff)).

THEOREM (NEC). Suppose that $f \in C^{\infty}\left(\mathbf{R}^{J}\right)$ is weakly continuous. Let

$$
E=\operatorname{span} \Lambda \subset \mathbf{R}^{J},
$$


let $d=\operatorname{dim} E$, and choose Cartesian coordinates in $\mathbf{R}^{J}$ so that $E=\left\{u \in \mathbf{R}^{J}: u_{d+1}\right.$ $\left.=\cdots=u_{J}=0\right\}$. Then

(i) The function $f(u)$ is a sum of polynomials in $u_{1}, \ldots, u_{d}$, homogeneous of degree at most $\min \{I, d\}$, with coefficients given by functions of $u_{d+1}, \ldots, u_{J}$ : i.e.,

$$
f(y)=\sum_{\alpha} c_{\alpha}\left(y_{d+1}, \ldots, y_{J}\right) P_{\alpha}\left(y_{1}, \ldots, y_{d}\right) .
$$

(ii) Each of the polynomials $P_{\alpha}$, homogeneous of degree $r \geq 2$, satisfies $L_{P_{\alpha}} \lambda_{1} \lambda_{2}$ $\cdots \lambda_{r}=0$, for every $\left(\lambda_{1}, \xi_{1}\right),\left(\lambda_{2}, \xi_{2}\right), \ldots,\left(\lambda_{r}, \xi_{r}\right) \in \mathscr{V}$ such that the rank of $\left(\xi_{1}, \ldots, \xi_{r}\right)$ is less than or equal to $r-1$. Here $L_{P_{\alpha}} \lambda_{1} \lambda_{2} \ldots \lambda_{r}$ is the action of the rth (Fréchet) derivative of $P_{\alpha}(y)$ on the collection of vectors $\left\{\lambda_{1}, \lambda_{2}, \ldots, \lambda_{r}\right\}$.

Since every polynomial $P$ homogeneous of degree $r$ can be written

$$
P(z)=\frac{1}{r !} L_{P} z \cdots z
$$

Theorem (Nec) provides a stronger condition than (L-H).

Of course, the question arises whether Theorem (Suff) (or something similar) provides a necessary condition. We are unaware of any weakly continuous function that does not satisfy this condition. (In fact, we are unaware of any that cannot be generated by wedge products.) The following remarks express what we can say about the generality and utility of our condition and its relation to the results mentioned above.

1. Part (ii) of Theorem (Nec) implies that the only interesting weakly continuous functions are polynomials.

2. It suffices to consider only polynomials that are homogeneous of some fixed degree in $U$ since it follows by rescaling that each term of a given homogeneity in an arbitrary weakly continuous polynomial must be weakly continuous as well.

3. Theorem (Suff) can be applied in general because every polynomial $P$ homogeneous of degree $L+1$ can be written as

$$
P(y)=P_{\mathrm{pol}}(y, y, \ldots, y)
$$

where $P_{\mathrm{pol}}\left(y_{0}, y_{1}, \ldots, y_{L}\right)$ is homogeneous of degree one in each slot. One way of doing this is in terms of the symmetric polarization which is given either in terms of the derivative as above, or by the formula

$$
P_{S}\left[U^{0}, \ldots, U^{L}\right] \equiv \frac{1}{2^{(L+1)}} \sum_{\sigma}(-1)^{\sigma} Q\left(\sum_{l=0}^{L} \sigma_{l} U^{l}\right) .
$$

Here $\sigma=\left(\sigma_{0}, \ldots, \sigma_{L}\right)$ is a sequence with values in $\{-1,1\}$, and $(-1)^{\sigma}=\sigma_{0} \sigma_{1} \cdots \sigma_{L}$. Note that it follows directly from this formula that

$$
P\left(U^{\varepsilon}\right) \stackrel{*}{\rightarrow} P(\bar{U}) \quad \text { in } L^{\infty}\left(\mathbf{R}^{I}\right)
$$

for every $U^{\varepsilon}$ satisfying (2.1)-(2.4) if and only if

$$
P_{S}\left[U^{0, \varepsilon}, \ldots, U^{L, \varepsilon}\right] \stackrel{*}{\rightarrow} P_{S}\left[\bar{U}^{0}, \ldots, \bar{U}^{L}\right]
$$

for every $U^{l, \varepsilon}$ satisfying $(2.1)-(2.4)$ separately, $l=0, \ldots, L$. Note also that $P_{S}$ can be written as the iterated composition of a sequence of quadratic maps. (This is easier to see from the derivative form of the polarization.) 
While this particular polarization of any polynomial is available (and thus can be used with our condition as a test for weak continuity) our theorem holds out the possibility of using other iterative maps. We are currently investigating whether or not our condition applied to the symmetric polarization is a necessary condition for weak continuity.

4. There are special cases in which we can show that our condition is necessary. In the case of a quadratic function of the form $Q\left(U^{1, \varepsilon}, U^{2, \varepsilon}\right)$, with $Q$ homogeneous of degree one in each slot, our condition (2.19) reads $Q\left(\lambda^{1}, \lambda^{2}\right)=0$ whenever $\xi \otimes \lambda^{1} \in \mathscr{V}_{1}$ and $\xi \otimes \lambda^{2} \in \mathscr{V}_{2}$ for the same $\xi$. Thus Theorem (Suff) reduces to Theorem (TA) when the polynomial $P$ is quadratic.

5. The following corollary states that Theorem (Suff) also reduces to condition (L-H) whenever the condition

$$
\lambda \otimes \xi \in \operatorname{span} \mathscr{W}_{l} \quad \text { if and only if } \quad \lambda \otimes \xi \in \mathscr{W}_{l}, \quad l=1, \ldots, L,
$$

is satisfied.

COROLLARY 2.1. If condition (2.3) holds, then condition (2.19) is equivalent to condition (L-H) holding for each $P^{l}, l=1, \ldots, L$; i.e.,

$$
P^{l}\left[v^{0}+t \lambda^{1}, \ldots, v^{l}+t \lambda^{l}\right] \text { is affine in } t
$$

for any $v^{n} \in \mathbf{R}^{J_{n}}, \lambda^{n} \in \Lambda_{n}$ such that $\xi \otimes \lambda^{n} \in V_{n}, n=0, \ldots, l$, for the same $\xi \in \mathbf{R}^{I}$.

PrOOF. The necessary condition ( $\mathrm{L}-\mathrm{H})$ is always implied by the sufficient condition (2.19), so we need only show that (2.24) implies (2.19) whenever condition (2.23) holds. Now (2.24) and the linearity of $P^{l}$ in each slot imply

$$
P^{l}\left[v^{0}, \ldots, v^{m-1}, \lambda^{m}, v^{m+1}, \ldots, v^{l-1}, \lambda^{l}\right]=0
$$

for every $v^{n} \in \mathbf{R}^{J_{n}}, n=0, \ldots, l-1$, and $\lambda^{m} \in \Lambda_{m}, \lambda^{l} \in \Lambda_{l}$ such that $\xi \otimes \lambda^{m} \in \mathscr{V}_{m}$, $\xi \otimes \lambda^{l} \in \mathscr{V}_{l}$ for the same $\xi \in \mathbf{R}^{I}$. Also, by the orthogonality hypothesis and (2.17) we have $\xi \otimes \bar{\lambda} \in \operatorname{span} \mathscr{W}_{l}$ if and only if

$$
\bar{\lambda}=P^{l-1}\left[v^{0}, \ldots, v^{m-1}, \lambda^{m}, v^{m+1}, \ldots, v^{l-1}\right]
$$

for some $v^{n} \in \mathbf{R}^{J_{n}}, n=0, \ldots, l-1$, and some $\lambda^{m} \in \Lambda_{m}$ such that $\xi \otimes \lambda^{m} \in \mathscr{V}^{m}$. But, by (2.25) if $\xi \otimes \lambda \in \mathscr{V}_{l}$ and $\xi \otimes \bar{\lambda} \in \operatorname{span} \mathscr{W}_{l}$ then

$$
\begin{aligned}
Q^{l}(\bar{\lambda}, \lambda) & =Q^{l}\left(P^{l-1}\left[v^{0}, \ldots, v^{m-1}, \lambda^{m}, v^{m+1}, \ldots, v^{l-1}\right], \lambda^{l}\right) \\
& \equiv P^{l}\left[v^{0}, \ldots, v^{m-1}, \lambda^{m}, v^{m+1}, \ldots, v^{l-1}, \lambda^{l}\right]=0,
\end{aligned}
$$

thus verifying (2.19).

6. We now note that the Wedge Product Theorem is a consequence of the previous corollary. To do this we identify the differential form $\omega_{l}^{\varepsilon}$ of degree $s_{l}$ with an element of $\mathbf{R}^{\left(\begin{array}{c}I \\ s_{l}\end{array}\right)}$ through its coefficients with respect to a fixed orthonormal basis. The iterated polynomials

$$
P^{l}: \mathbf{R}^{\left(\begin{array}{c}
I \\
s_{0}
\end{array}\right)} \times \cdots \times \mathbf{R}^{\left(\begin{array}{c}
I \\
s_{l-1}
\end{array}\right)} \rightarrow \mathbf{R}^{\left(\begin{array}{c}
s_{0}+\cdots+s_{l-1} \\
I
\end{array}\right)}
$$

are defined (with an obvious abuse of notation) by

$$
P^{l}\left[\omega_{0}, \ldots, \omega_{l-1}\right]=\omega_{0} \wedge \cdots \wedge \omega_{l-1} .
$$


It follows from $(2.15),(2.17),(2.18)$, and the fact that the wedge product is the symbol for the exterior derivative operator that

$$
\mathscr{V}_{l}=\left\{(\xi, \lambda) \in \mathbf{R}^{I} \times \mathbf{R}^{\left(\begin{array}{c}
I \\
s_{l}
\end{array}\right)}: \xi \wedge \lambda=0\right\}, \quad l=0, \ldots, L,
$$

and

$$
\mathscr{W}_{l}=\left\{(\xi, \lambda) \in \mathbf{R}^{I} \times \mathbf{R}^{\left(s_{0}+\cdots+s_{l-1}\right)}: \xi \wedge \lambda=0\right\}, \quad l=1, \ldots, L .
$$

Our result follows from (2.27) and Corollary 2.1.

7. Finally, we comment that in the proof below it will be apparent that Theorem (Suff) is sharp in the sense that we completely characterize (in Lemma 3.2 ) the constraints under which the iterated polynomials can oscillate.

3. Proof of Theorem (Suff). In this section we prove Theorem (Suff). Our method is to construct conditions under which we can iterate the quadratic theorem of Tartar.

We introduce some new notation. Let $U^{\varepsilon}$ denote any sequence of functions satisfying (2.1)-(2.4). For each $k=1, \ldots, K$, let $A^{k}$ denote the $I \times J$ matrix $A_{i j}^{k}$ constructed from the constraints in (2.3). Let $\langle A, B\rangle$ denote the componentwise inner product between arbitrary $I \times J$ matrices $A$ and $B$, so that

$$
\langle A, B\rangle=\operatorname{Trace}\left(A^{\mathrm{tr}} B\right)=\sum_{i, j} A_{i j} B_{i j} .
$$

With this notation the set $\mathscr{V}$ can be defined by

$$
\mathscr{V}=\left\{\xi \otimes \lambda \in \mathscr{L}\left(\mathbf{R}^{J}, \mathbf{R}^{I}\right):\left\langle A^{k}, \xi \otimes \lambda\right\rangle=0 \text { for each } k=1, \ldots, K\right\},
$$

and we can rewrite (2.3) as

$$
\operatorname{div}\left(A^{K} U^{\varepsilon}\right) \in \text { compact set in } H_{\text {loc }}^{-1}\left(\mathbf{R}^{I}\right), \quad k=1, \ldots, K,
$$

where $\operatorname{div} \vec{V}$ denotes the divergence in $x$ of the vector field $\vec{V} \in \mathbf{R}^{J}$.

The following lemma characterizes the first-order linear differential operators on $U^{\varepsilon}$ that lie in a compact set in $H_{\text {loc }}^{-1}\left(\mathbf{R}^{I}\right)$ as a consequence of (3.3).

LEMMA (3.1). An $I \times J$ constant matrix $B$ satisfies the condition

$$
\operatorname{div}\left(B U^{\varepsilon}\right) \in \text { compact set in } H_{\mathrm{loc}}^{-1}\left(\mathbf{R}^{I}\right)
$$

for all $U^{\varepsilon}$ satisfying (2.1)-(2.4) if and only if

$$
\langle B, \xi \otimes \lambda\rangle=0
$$

for all $\xi \otimes \lambda \in \mathscr{V}$.

We postpone the proof until after the proof of Theorem (Suff). To this end, assume that $(2.1)-(2.4)$ has the decomposition $(2.1)_{l}-(2.4)_{l}$ for $l=0, \ldots, L$, and let $P^{L}$ denote any polynomial generated by $(2.14)$. Let

$$
Z^{l, \varepsilon}: \mathbf{R}^{I} \rightarrow \mathbf{R}^{M_{l}}, \quad l=1, \ldots, L,
$$

denote the function

$$
Z^{l, \varepsilon}(x)= \begin{cases}u^{0, \varepsilon}(x), & l=1, \\ P^{l-1}\left[u^{0, \varepsilon}(x), \ldots, u^{l-1, \varepsilon}(x)\right], & l=2, \ldots, L .\end{cases}
$$

In order to iterate the quadratic theorem, we characterize the derivatives of $Z^{l, \varepsilon}$ that are bounded as a consequence of $U^{\varepsilon}$ satisfying $(2.1)_{l}-(2.4)_{l}$. 
Lemma (3.2). Fix $l, 1 \leq l \leq L$. An $I \times M_{l}$ matrix $D$ satisfies the condition

$$
\operatorname{div}\left(D Z^{l, \varepsilon}\right) \in \text { compact set in } H_{\mathrm{loc}}^{-1}\left(\mathbf{R}^{I}\right)
$$

for all $U^{\varepsilon}$ satisfying $(2.1)_{m}-(2.4)_{m} m=0, \ldots, l-1$, if and only if $D \in \mathscr{W}_{l}^{\perp}$; i.e.,

$$
\langle D, \xi \otimes \bar{\lambda}\rangle=0
$$

for all $\xi \otimes \bar{\lambda} \in \mathscr{W}_{l}$, where $\mathscr{W}_{l}$ is defined in (2.18).

PROOF. We first show that (3.10) implies (3.11). Assume for contradiction that there exists an $I \times M_{l}$ matrix $D$ such that (3.10) holds and $\xi \otimes \bar{\lambda} \in \mathscr{W}_{l}$ such that $\langle D, \xi \otimes \bar{\lambda}\rangle \neq 0$. Then by $(2.17)$, there exist $m, 0 \leq m \leq l-1 ; \xi \otimes \lambda^{m} \in \mathscr{V}_{m}$; $v^{n} \in \mathbf{R}^{J_{n}}, n=0, \ldots, l-1$; such that

$$
\bar{\lambda}=P^{l-1}\left[v^{0}, \ldots, v^{m-1}, \lambda^{m}, v^{m+1}, \ldots, v^{l-1}\right] .
$$

Thus, for $0 \leq n \leq l-1$, define

$$
u^{n, \varepsilon}= \begin{cases}v^{n} & \text { if } n \neq m, \\ \lambda^{m} \sin \left(\frac{x \cdot \xi}{\varepsilon}\right) & \text { if } n=m .\end{cases}
$$

Clearly $U^{\varepsilon}$ satisfies $(2.1)_{n}-(2.4)_{n}$ for $0 \leq n \leq l-1$. Since $P^{l}$ is linear in each component, we have in this case that

$$
\begin{aligned}
Z^{l, \varepsilon} & =P^{l-1}\left[u_{1}^{\varepsilon}, \ldots, u_{l-1}^{\varepsilon}\right] \\
& =P^{l-1}\left[v^{0}, \ldots, v^{m-1}, \lambda^{m} \sin \left(\frac{x \cdot \xi}{\varepsilon}\right), v^{m+1}, \ldots, v_{l-1}\right] \\
& =\bar{\lambda} \sin \left(\frac{x \cdot \xi}{\varepsilon}\right)
\end{aligned}
$$

where $\bar{\lambda}$ is given by (3.12). Thus by (3.11),

$$
\begin{aligned}
\operatorname{div} D Z^{l, \varepsilon} & =\left\{D \cdot \bar{\lambda} \sin \left(\frac{x \cdot \xi}{\varepsilon}\right)\right\} \\
& =\frac{1}{\varepsilon} \sum_{i, j} D_{i j} \bar{\lambda}_{j} \xi_{i} \cos \left(\frac{x \cdot \xi}{\varepsilon}\right) \\
& =\frac{1}{\varepsilon} \cos \left(\frac{x \cdot \xi}{\varepsilon}\right)\langle D, \xi \otimes \bar{\lambda}\rangle \neq 0,
\end{aligned}
$$

and so no subsequence of $\operatorname{div} D Z^{l, \varepsilon}$ lies in a compact set in $H_{\text {loc }}^{-1}\left(\mathbf{R}^{I}\right)$ as $\varepsilon \rightarrow 0$, a contradiction. Thus (3.10) implies (3.11).

Conversely, assume (3.11) holds for some $I \times M_{l}$ matrix $D$, and let $U^{\varepsilon}$ be an arbitrary sequence satisfying (2.1)-(2.4). For simplicity we drop the index $\varepsilon$ in this part of the proof. Thus we can write

$$
\operatorname{div} D Z^{l}(x)=\sum_{i} \frac{\partial}{\partial x_{i}} \sum_{j} D_{i j} P_{j}^{l-1}\left[u^{0}(x), \ldots, u^{l-1}(x)\right]
$$

where $i$ is summed from 1 to $I$ and $j$ is summed from 1 to $M_{l}$. We claim that to show (3.10) holds one need only analyze terms of the form:

$$
\sum_{i, j} D_{i j} P_{j}^{l-1}\left[e^{\sigma_{0}}, \ldots, e^{\sigma_{m-1}}, \frac{\partial u^{m}}{\partial x_{i}}, e^{\sigma_{m+1}}, \ldots, e^{\sigma_{l-1}}\right]
$$


where the $e^{\sigma_{n}}$ are fixed basis elements of $\mathbf{R}^{J_{n}}$. To see this note that by the multilinearity of $P_{j}^{l-1}$ the differentiations in (3.12) can be carried out using Leibniz rule yielding

$\operatorname{div} D Z^{l}=\sum_{i, j} D_{i j}\left(P_{j}^{l-1}\left[\frac{\partial u^{0}}{\partial x_{i}}, u^{1}, \ldots, u^{l-1}\right]+\cdots+P_{j}^{l-1}\left[u^{0}, u^{1}, \ldots, \frac{\partial u^{l-1}}{\partial x_{i}}\right]\right)$.

The undifferentiated factors can be written as

$$
u^{n}(x)=\sum_{\sigma_{n}} u_{\sigma_{n}}^{n}(x) e^{\sigma_{n}}
$$

when $\sigma_{n}$ is summed from 1 to $J_{n} ; e_{\sigma_{n}}$ are fixed basis elements in $\mathbf{R}^{J_{n}}$; and $u_{\sigma_{n}}^{n}(x)$ are real valued functions, uniformly bounded in $L^{\infty}\left(\mathbf{R}^{I}\right)$. Again using the multilinearity of $P_{j}^{l-1}$, we see that $\operatorname{div} D Z^{l}$ is the sum of products of $L^{\infty}$ functions with factors of the form (3.13).

Thus to verify (3.10) it suffices to show that

$$
\sum_{i j} D_{i j} P_{j}^{l-1}\left[v^{0}, \ldots, v^{m-1}, \frac{\partial u^{m}}{\partial x_{i}}, v^{m+1}, \ldots, v^{l-1}\right] \in \text { compact set in } H_{\mathrm{loc}}^{-1}\left(\mathbf{R}^{I}\right)
$$

for arbitrary $v^{n} \in \mathbf{R}^{J_{n}}$. Since $P_{j}^{l-1}$ is linear in the $m$ th slot, there is an $M_{l} \times J_{m}$ matrix $A$ depending on $P^{l-1}$ and the $v^{n}$ such that

$$
P_{j}^{l-1}\left[v^{0}, \ldots, v^{m-1}, \frac{\partial u^{m}}{\partial x_{i}}, v^{m+1}, \ldots, v^{l-1}\right]=A \frac{\partial u^{m}}{\partial x_{i}}=\frac{\partial}{\partial x_{i}}\left(A u^{m}\right) .
$$

Hence

$$
\sum_{i j} D_{i j} P_{j}^{l-1}\left[v^{0}, \ldots, v^{m}, \frac{\partial u^{m}}{\partial x_{i}}, v^{m+1}, \ldots, v^{l-1}\right]=\operatorname{div}\left(D A u^{m}\right) .
$$

But by Lemma (3.1),

$$
\operatorname{div}\left(D A u^{m}\right) \in \text { compact set in } H_{\text {loc }}^{-1}\left(\mathbf{R}^{I}\right)
$$

for all $u^{m}$ satisfying $(2.1)_{m}(2.4)_{m}$ if and only if

$$
\langle D A, \xi \otimes \lambda\rangle=0
$$

for all $\xi \otimes \lambda \in \Lambda_{m}$. But,

$$
\langle D A, \xi \otimes \lambda\rangle=\langle D, \xi \otimes(A \lambda)\rangle
$$

and by the definition of $A$

$$
A \lambda=P^{l-1}\left[v^{0}, \ldots, v^{m-1}, \lambda, v^{m-1}, \ldots, v^{l-1}\right],
$$

so by $(2.17) \xi \otimes(A \lambda) \in \mathscr{W}_{l}$. Statement (3.17) follows at once from (3.10). The proof of Lemma (3.2) is complete.

Proof OF THEOREM (SUFF). Now that we have characterized the possible oscillations of our iterated polynomials Theorem (Suff) follows directly: Let $U^{\varepsilon}-$ $\bar{U}$ be any sequence satisfying $(2.1)-(2.4)$ and having a decomposition $\left(u^{0, \varepsilon}, \ldots, u^{L, \varepsilon}\right)$

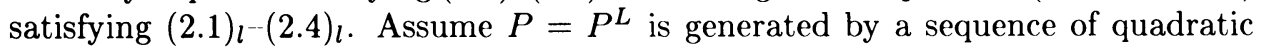


maps via (2.14), and suppose condition (2.19) of Theorem (Suff) holds. We now show by induction on $l$ that

$$
P\left[u^{0, \varepsilon}, \ldots, u^{L, \varepsilon}\right] \stackrel{*}{\rightarrow} P\left[\bar{u}^{0}, \ldots, \bar{u}^{L}\right] \quad \text { in } L^{\infty}\left(\mathbf{R}^{I}\right) .
$$

Case $(l=1)$. Recall that $P^{1}\left[u^{0}, u^{1}\right]=Q^{1}\left(u^{0}, u^{1}\right)$ and that $\mathscr{W}_{1}=\mathscr{V}_{0}$. Theorem (TA) says that $Q^{1}\left(u^{0, \varepsilon}, u^{1, \varepsilon}\right) \stackrel{*}{\rightarrow} Q^{1}\left(\bar{u}^{0}, \bar{u}^{1}\right)$ if and only if $Q^{1}(\bar{\lambda}, \lambda)=0$ whenever $\xi \otimes \bar{\lambda} \in \mathscr{V}_{0}$ and $\xi \otimes \lambda \in \mathscr{V}_{1}$ for the same $\xi \in \mathbf{R}^{I}$. However, we observe that $\xi \otimes \bar{\lambda} \in \mathscr{V}_{0}$ if and only if $\xi \otimes \bar{\lambda} \in \operatorname{span} \mathscr{V}_{0}=\operatorname{span} \bar{W}$, so by Theorem (TA)

$$
P^{1}\left[u^{0, \varepsilon}, u^{1, \varepsilon}\right] \stackrel{*}{\rightarrow} P^{1}\left[\bar{u}^{0}, \bar{u}^{1}\right], \quad \text { in } L^{\infty}\left(\mathbf{R}^{I}\right),
$$

or in different notation

$$
Z^{2, \varepsilon} \stackrel{*}{\rightarrow} \bar{Z}^{2} \equiv P^{1}\left[\bar{u}^{0}, \bar{u}^{1}\right] \quad \text { in } L^{\infty}\left(\mathbf{R}^{I}\right) .
$$

Case $(1<l \leq L)$. We now assume

$$
Z^{l, \varepsilon} \stackrel{*}{\rightarrow} \bar{Z}^{l} \equiv P^{l-1}\left[\bar{u}^{0}, \ldots, u^{l-1}\right] \quad \text { in } L^{\infty}\left(\mathbf{R}^{I}\right),
$$

and show that

$$
Z^{l+1, \varepsilon} \stackrel{*}{\rightarrow} \bar{Z}^{l+1} \quad \text { in } L^{\infty}\left(\mathbf{R}^{I}\right) .
$$

Once again we use the quadratic theorem and the representation

$$
Z^{l+1, \varepsilon}=P^{l}\left[u^{0, \varepsilon}, \ldots, u^{l, \varepsilon}\right] \equiv Q^{l}\left(Z^{l, \varepsilon}, u^{l, \varepsilon}\right) ;
$$

but now we need to use Lemma 3.2 and the information that $Z^{l, \varepsilon}$ satisfies the first-order differential constraints

$$
\operatorname{div}\left(D Z^{l, \varepsilon}\right) \in H_{\mathrm{loc}}^{-1}\left(\mathbf{R}^{I}\right)
$$

for every $D \in \mathscr{W}_{l}{ }^{\perp}$. The characteristic set for these constraints is defined to be

$$
\begin{aligned}
\hat{V}_{l} & \equiv\left\{\xi \otimes \lambda \in \mathscr{L}\left(\mathbf{R}^{M_{l}}, \mathbf{R}^{I}\right):\langle\xi \otimes \lambda, D\rangle=0, \forall D \in \mathscr{W}_{l}^{\perp}\right\} \\
& =\left\{\text { rank-1 matrices in } \mathscr{W}_{l}^{\perp \perp}\right\} \\
& =\left\{\text { rank-1 matrices in span } \mathscr{W}_{l}\right\} .
\end{aligned}
$$

Thus, by Theorem (TA) $Q^{l}\left(Z^{l, \varepsilon}, u^{l, \varepsilon}\right) \stackrel{*}{*} Q^{l}\left(\bar{Z}^{l}, \bar{u}^{l}\right)$ (i.e., (3.22) holds) if and only if $Q^{l}(\bar{\lambda}, \lambda)=0$ whenever $\xi \otimes \bar{\lambda} \in \mathscr{V}_{l}$ and $\xi \otimes \lambda \in \mathscr{V}_{l}$ for the same $\xi \in \mathbf{R}^{I}$. But by (3.24) this is just condition (2.19), so the induction is completed and Theorem (Suff) is proved.

Note that Lemma (3.2) indicates the sense in which Theorem (Suff) is the best sufficient condition that only takes account of bounds on derivatives, and not more subtle constraints imposed on $U^{\varepsilon}$ by polynomials of type (2.14); i.e., Lemma (3.2) gives necessary and sufficient conditions for derivatives of $Z^{l, \varepsilon}$ to be bounded in $H_{\text {loc }}^{-1}$; and Theorem (Suff) is based on Theorem (TA) which is the best condition for weak continuity of quadratic function that only takes account of bounds or derivatives.

PROOF OF LEMMA (3.1). Assume first that (3.4) holds and note that if (3.5) fails, then there exists $\xi \otimes \lambda \in \mathscr{V}$ such that

$$
\langle B, \xi \otimes \lambda\rangle \neq 0 \text {. }
$$


But then, as we noted before in different notation,

$$
U^{\varepsilon}(x)=\lambda \sin \left(\frac{x \cdot \xi}{\varepsilon}\right)
$$

satisfies (2.1)-(2.4), while

$$
\operatorname{div}\left(B U^{\varepsilon}\right)=\operatorname{div} B \lambda \sin \left(\frac{x \cdot \xi}{\varepsilon}\right)=\frac{1}{\varepsilon}\langle B, \xi \otimes \lambda\rangle \cos \left(\frac{x \cdot \xi}{\varepsilon}\right)
$$

which in light of (3.25) is not compact in $H_{\mathrm{loc}}^{-1}$ for any subsequence. Thus (3.4) implies (3.5).

Conversely, assume that (3.5) holds. In order to show (3.4) it suffices to assume that

$$
U^{\varepsilon} \stackrel{*}{\rightarrow} 0 \text { in } L^{\infty}\left(\mathbf{R}^{I}\right)
$$

and

$$
\operatorname{Supp} U^{\varepsilon} \subseteq K
$$

for $K \subset \mathbf{R}^{I}$ compact, and prove that at least for a subsequence

$$
\operatorname{div} B U^{\varepsilon} \rightarrow 0 \quad \text { in } H^{-1}\left(\mathbf{R}^{I}\right) \text {. }
$$

This simplification is the result of standard localization techniques.

Writing (3.5) in component form gives $\sum_{i, j} B_{i j} \xi_{i} \lambda_{j}=0$ where $i$ is summed from 1 to $I, j$ from 1 to $J$. This implies that for every $\varepsilon>0$ there exists $C_{\varepsilon}>0$ such that

$$
\left|\sum_{i, j} B_{i j} \xi_{i} \lambda_{j}\right|^{2} \leq C_{\varepsilon} \sum_{k=1}^{K}\left|\sum_{i, j} A_{i j}^{k} \xi_{i} \lambda_{i}\right|^{2}+\varepsilon|\xi|^{2}|\lambda|^{2} l
$$

for every $\xi \in \mathbf{R}^{I}, \lambda \in R^{J}$. Indeed, (3.29) holds on the compact set $|\xi|=|\lambda|=1$ because the left-hand side of (3.29) vanishes whenever $\sum_{k=1}^{K}\left|\sum_{i, j} A_{i j}^{k} \xi_{i} \lambda_{i}\right|^{2}$ vanishes. Thus (3.29) extends to all $\xi \in \mathbf{R}^{I}, \lambda \in \mathbf{R}^{J}$ since both sides of (3.29) are homogeneous of degree two in $\xi$ and $\lambda$. Replacing $|\xi|^{2}$ by $1+|\xi|^{2}$ on the right-hand side of (3.29) gives

$$
\frac{\left|\sum_{i, j} B_{i j} \xi_{i} \lambda_{j}\right|^{2}}{1+|\xi|^{2}} \leq C_{\varepsilon} \sum_{k=1}^{K} \frac{\left|\sum_{i, j} A_{i j}^{k} \xi_{i} \lambda_{j}\right|^{2}}{1+|\xi|^{2}}+\varepsilon|\lambda|^{2}
$$

for all $\xi \in \mathbf{R}^{I}, \lambda \in \mathbf{R}^{J}$. Substituting $\hat{U}^{\varepsilon}(\xi)$ (the Fourier transform of $U^{\varepsilon} \in L^{2}$ ) for $\lambda$ in (3.30) and integrating over $\xi \in \mathbf{R}^{I}$ gives

$$
\left\|\operatorname{div} B U^{\varepsilon}\right\|_{H_{-1}}^{2} \leq C^{\varepsilon} \sum_{k=1}^{K}\left\|\operatorname{div} A^{k} U^{\varepsilon}\right\|_{H^{-1}}^{2}+\varepsilon\left\|U^{\varepsilon}\right\|_{L^{2}}^{2},
$$

where we have applied Parseval's identity

$$
\left\|U^{\varepsilon}\right\|_{L^{2}}^{2}=\left\|\hat{U}^{\varepsilon}\right\|_{L^{2}}^{2}
$$


But by (2.3), (3.26) and uniqueness of weak limits, there exists a subsequence of $\left\{U^{\varepsilon}\right\}$ such that

$$
C^{\varepsilon} \sum_{k=1}^{K}\left\|\operatorname{div} A^{k} U^{\varepsilon}\right\|_{H^{-1}}^{2} \rightarrow 0 ;
$$

and by (2.2), (3.27), $\left\|U^{\varepsilon}\right\|_{L^{2}}^{2}$ is uniformly bounded. Thus (3.28) follows at once from (3.31), and the lemma is proved.

\section{REFERENCES}

1. B. Dacorogna, Weak continuity and weak lower semi-continuity of nonlinear functionals, Lecture Notes in Math., vol. 922, Springer-Verlag, Berlin and New York, 1982.

2. C. B. Morrey, Multiple integrals in the calculus of variations, Springer-Verlag, Berlin and New York, 1966.

3. F. Murat, Compacité par compensation: condition nécessaire et suffisante de continuité faible sous une hypothèse de rang constant, Ann. Scuola Norm. Sup. Pisa 8 (1981), 69-102.

4. J. Robbin, R. C. Rogers, and B. Temple, On weak continuity and the Hodge decomposition, Trans. Amer. Math. Soc. 303 (1987), 1-10.

5. L. Tartar, Compensated compactness and applications to partial differential equations, Nonlinear Analysis and Mechanics: Heriot-Watt Symposium 4 (R. J. Knops, ed.), Pitman, 1979.

6. __ The compensated compactness method applied to systems of conservation laws, Systems of Nonlinear Partial Differential Equations (J. M. Ball, ed.), NATO AS1 Series, Reidel, 1983.

7. __ Oscillations in nonlinear partial differential equations, Lectures in Applied Math., vol. 23, Amer. Math. Soc., Providence, R.I., 1986.

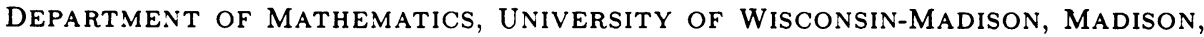
WISCONSIN 53706

Department of Mathematics, University of California at DaVis, Davis, CalIFORNIA 95616 (Current address of Blake Temple)

Current address (R. C. Rogers): Department of Mathematics, Virginia Polytechnic Institute and State University, Blacksburg. Virginia 24060 